\title{
Review of: "Compositional analysis of ruminal bacteria from ewes selected for somatic cell score and milk persistency"
}

Renee Petri

Potential competing interests: The author(s) declared that no potential competing interests exist.

This research article looks at the impact of genetic selection of ewes for somatic cell counts or milk persistency and the relationships to rumen bacteria using compositional analysis.

This paper is extremely well written and does a excellent job of explaining the concept of compositional analysis to the reader (as many people are unaware of this statistical analysis and the inaccuracies of using typical statistical analysis in microbial ecosystem data).

I have a few points that would be beneficial to the readers for the authors to clarify in the material and methodology (prior to the statistical model explanation).

1. That this is a multi-year study and how many samples were taken each year

2. The age range of the ewes (maybe also primiparous vs. multiparous - since this is a known factor effecting SCS/milk production/mastitis/ and milk composition)

3. Did the authors run a negative control in the sequencing that allows comparison of all sequencing runs.

4. Why rare OTUs were removed as previous research has shown that rare OTUs may correlate with production traits such as methane and feed efficiency (in cows). 